\title{
Pyoderma Gangcrenosum in a Breast Cancer Patient: Report of a Case and Review of the Literature
}

\author{
F. Peeters ${ }^{1}$, X.B. $\operatorname{Trinh}^{1}$, L. Verkinderen ${ }^{1}$, L. Dirix ${ }^{2}$ and P. Van Dam ${ }^{* 1}$ \\ ${ }^{I}$ Department of Obstetrics, Gynaecology and Gynaecological Oncology, Sint Augustinus Hospital, Antwerp, Belgium \\ ${ }^{2}$ Cancer Center, Sint Augustinus Hospital, Antwerp, Belgium
}

\begin{abstract}
Pyoderma gangrenosum (PG) is a primarily sterile neutrophilic dermatosis. It starts spontaneously or due to pathergy $(25-50 \%)$ with very painful ulcers with bluish borders, which grow rapidly and give expanding tissue necrosis. General incidence is estimated at 1/100000 per year. In this report a breast cancer patient developed PG due to surgical correction of a retracted scar of a lumpectomy. Diagnosis was made after her apparent inflammatory breast did not respond to broad spectrum antibiotics and became rapidly necrotic. This remained limited to the area of prior radiotherapy. Good response to systemic corticosteroids confirmed diagnosis of PG.
\end{abstract}

Keywords: Pyoderma gangrenosum, neutrophilic dermatosis, pathergy, pyoderma gangrenosum in a breast cancer patient: report of a case and review of the literature.

\section{INTRODUCTION}

Pyoderma gangrenosum (PG) was first described by Brocq in 1916 [1] and later named by Brunsting [2] in 1930. PG is a primarily sterile neutrophilic dermatosis. It starts with very painful ulcers with rapid growth and expanding tissue necrosis. Ulcers are undermined with bluish borders and surrounded with erythema. PG occurs worldwide and it equally affects both genders. The peak of incidence occurs between the ages of 30 to 50 years. Because accurate general data are missing general incidence is estimated at 1/100000 per year [3]. In 25 to $50 \%$ an non specific, external stimulus provokes PG [4]. This is called pathergy. Surgery, an injection, an insect sting and other forms of trauma are known external triggers of PG. However, mostly these ulcers develop spontaneously. Preferably PG is located pretibial but also affects other sides of the skin. Sometimes other organs can be affected (for example sterile neutrophilic lung ulcers, genital mucosa, spleen infiltrates, etc.) [5]. In present report, we discuss a post-radiation/post-surgery case of PG of the breast.

\section{CASE REPORT}

A 51-year-old menopausal woman was diagnosed with a pT1cpN0(sn)M0 poorly differentiated breast adenocarcinoma in her right breast in January 2007. Histology showed no lymphovascular invasion, free margins and a high mitotic activity index. The tumour was proven to be immunohistochemically triple negative: no immunoreactivity for oestrogen receptors, neither for progesterone receptors or Her2/neu-oncogen protein. Her adjuvant treatment consisted of chemotherapy and radiotherapy. She received four courses of cyclophosphamide $1000 \mathrm{mg} / \mathrm{m}^{2}$, epirubicine $500 \mathrm{mg} / \mathrm{m}^{2}$ and 5 -fluorouracil $1000 \mathrm{mg} / \mathrm{m}^{2}$ followed by four courses of

*Address correspondence to this author at the St. Augustinus Hospital, Oosterveldlaan 24, 2650 Wilrijk-Antwerp, Belgium; Tel: +32 344337 37; E-mail: peter.vandam@gza.be docetaxel $100 \mathrm{mg} / \mathrm{m}^{2}$. Afterwards, she underwent radiotherapy of the right chest wall with a total dose of 50 Gray given in 25 fractions followed by a boost of 16 Gray in 8 fractions on the tumour bed. The radiotherapy treatment was well tolerated besides local erythema of the irradiated skin grade 2.

One year after initial diagnosis, the patient wanted a surgical correction of her lumpectomy scar which was slightly retracted. Twelve days after the repair of her scar, she presented at the emergency unit with a painful, swollen, partially red breast (only at the previously irradiated area) and a subfebrile temperature since 5 days. Two days earlier her general practitioner started her on amoxiclavulanic acid $500 \mathrm{mg}$ orally (po) 3 times a day.

Upon arrival in the emergency she had a temperature of $38.8^{\circ} \mathrm{C}$ to $39.5^{\circ} \mathrm{C}$ and a completely red breast. Intravenous (IV) treatment with amoxiclavulanic acid $3 \times 2 \mathrm{~g}$, amykacine $1 \mathrm{~g}$ and clindamycine $3 \times 600 \mathrm{mg}$ daily was started. As there was no clinical improvement, the wound was opened and rinsed with antiseptic and oxygenated water twice daily. Although there was the impression of sepsis, all hemocultures remained negative. After 72 hours IV antibiotics were changed to piperacilline $4 \mathrm{~g}$ and tazobactam $500 \mathrm{mg} 3$ times daily in association with clarithromycine $500 \mathrm{mg}$ twice daily. Fluconazole $200 \mathrm{mg}$ IV was also added. The patient was stable but remained to have the same redness of her right breast as well as spikes of fever.

Six days after admission, 2 small pustules appeared just above the reopened scar. The next day two third of the skin of her right breast became necrotic in a few hours time $(10 \mathrm{~cm}$ in diameter) (Fig. 1). All necrotic debris, which was only superficial, was removed surgically. Cultures of the surgically removed tissue remained sterile. Clinically a diagnosis of PG was made. Histology showed a diffuse neutrophilic dermatosis with some vasculitis and confirmed the diagnosis supporting the presence of PG by exclusion. Because inflammatory bowel diseases and hematologic 
malignancies often are associated with PG, several screening investigations were performed. Fortunately they were all reassuring. Antibiotics IV were ceased and prednisone 40 $\mathrm{mg}$ twice daily was started. In 48 hours there was a spectacular regression of the redness. Because of the large skin defect patient was transferred to a specialized Dermatology unit in the University Hospital Leuven.

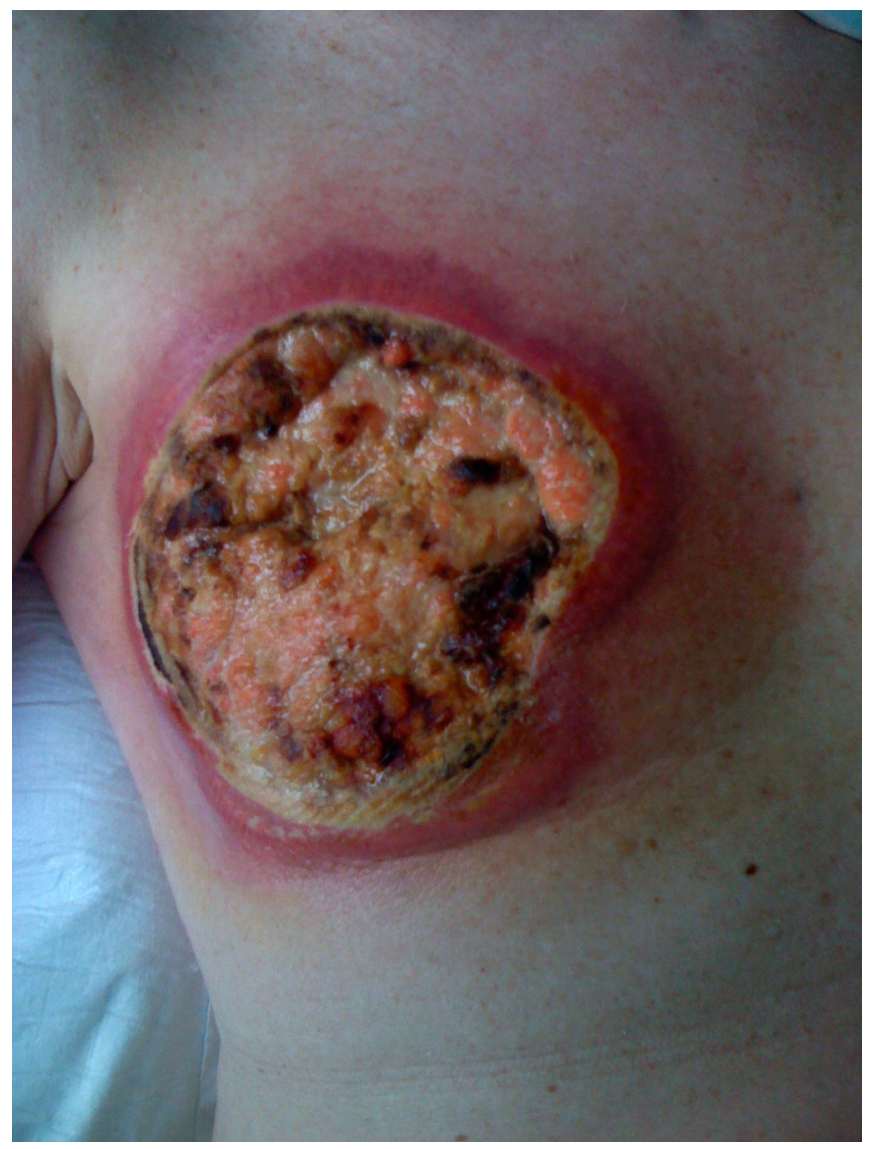

Fig. (1). Two third of the skin of her right mamma became necrotic in a few hours time: pyoderma gangrenosum.

Topical wound care consisted of hydrating gauzes, bandages with silicones and zinc paste, while systemic prednisone was continued and slowly reduced under protection of the stomach with ranitidine. One month after initial presentation at the emergency she was discharged home. It took several months for the skin defect to heal up (Fig. 2).

One year after the diagnosis of PG she presented on ultrasound with two pathological enlarged infraclavicular lymph nodes. Further investigations showed multiple metastasized lesions to liver, lower mediastinum, right pelvis and head of right femur as well as to several thoracic and lumbar corpora vertebra. She is receiving palliative chemotherapy (Fig. 3).

\section{DISCUSSION}

The aetiology and pathogenesis of PG are not yet identified. Nearly $50 \%$ of patients have an underlying disorder, of which inflammatory bowel disease (IBD) such as ulcerative colitis and Crohn's disease, occurs often (1015\%) [4] But also different types of arthritis, hemotologic and liver diseases as well as cancer, systemic lupus erythomatosis, sarcoidosis, HIV, pregnancy [6, 7] appear mostly before, but also during or after the outbreak of PG. When arthritis is associated, PG-ulcers only heal in $23.4 \%$ compared to $78.9 \%$ without arthritis [8]. There is no pathognomonic or specific histology. The most common feature of PG on histology is a diffuse neutrophilic dermatosis. This is because histology changes with the stage of PG. Early biopsy shows a dermal neutrophilic abscess, later progressing to epidermal necrosis and superficial ulceration with dermal oedema $[9,10]$.

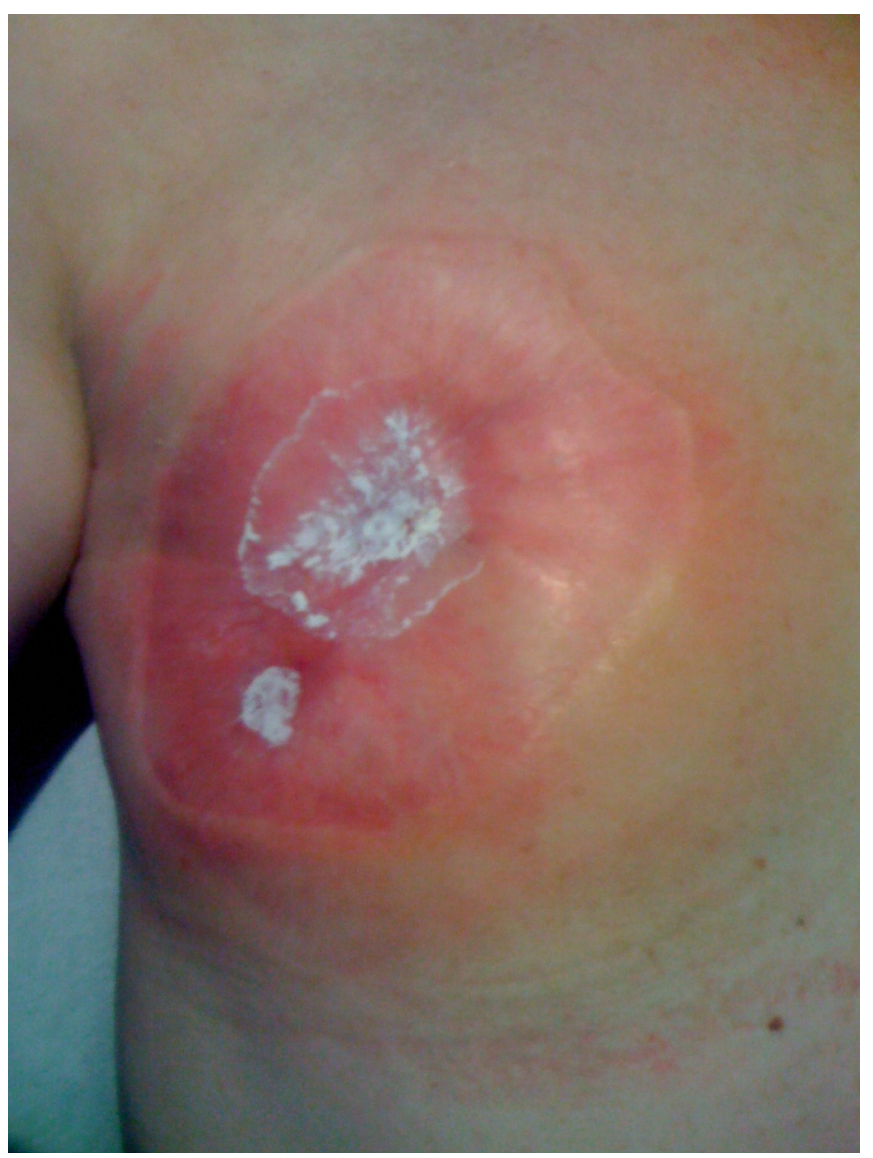

Fig. (2). After several months the skin defect due to pyoderma gangrenosum was healed.

Diagnosis is made clinically. Patients present with an erythematous papule or pustule that breaks down to form an ulcer with purulent discharge, violaceous coloured borders. Signs are unsupportable pain, fever (up to $40^{\circ}$ Celsius) and malaise. Classic wound care and antibiotics are ineffective. Blood and wound cultures remain negative. Although a biopsy can exacerbate the condition, it is more important to rule out other conditions. Treatment of PG can adversely affect other causes of ulceration and its therapy produces substantial side effect [10]. Reconsideration of the diagnosis of PG is necessary when standard therapy fails.

To rule out other conditions - such as vasculitis, infections, malignancies, drug reactions - blood tests (blood count, serum electrolytes, chemistry studies, immunoelectrophoresis, serology (syphilis, hepatitis, HIV), titers of antinuclear antibodies, rheumatoid factor, antiphospholipid antibody, antineutrophilic cytoplasmic antibodies), urine analysis, chest X-ray, abdominal ultrasound, bone marrow 
biopsy, rectoscopy and colonoscopy, biopsy and cultures can be useful [11].

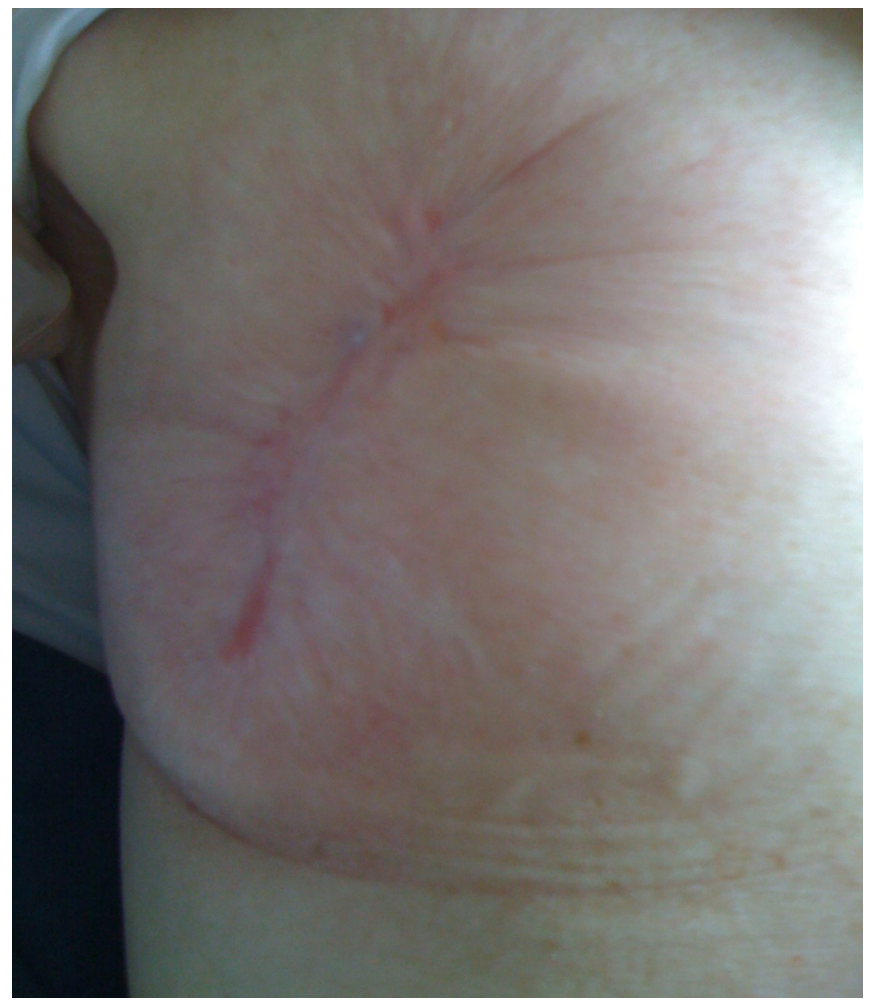

Fig. (3). One year after the diagnosis of pyoderma gangrenosum.
PG is classified into 4 clinical classes [12] (our patient had ulcerative $\mathrm{PG}$ ):

1. Ulcerative $\mathrm{PG}$ (classic form of $\mathrm{PG}, 70 \%$ has an associated disease)

2. Pustular PG (pustules do not evolve into ulcers; mostly associated with IBD)

3. Bullous PG (superficial painful bulla evolve into erosion and superficial ulcers; often associated with myelodysplastic disease)

4. Vegetative PG (chronic lesions, limited and nonaggressive; no systemic disease associated).

Medical treatment consists of systemic and topical therapy and pain relief. Systemic therapy is reviewed and summarized in Table 1 [5, 13, 14]. Conservative local wound management is necessary. One should be careful with surgery as it can be the trigger of PG (pathergy). Split skin grafts have variable outcomes [15]. The cornerstone is moist wound management with dressings of different layers: from hydration (hydrogel with corticoids) to absorbing (alginate dressing). Successes are also booked with topical $0.5 \%$ tacrolimus, $\quad 10 \% \quad 5$-aminosalicylic acid cream and cyclosporine A $35 \mathrm{mg}$ (in isotonic saline) locally injected $[5$, $13,14]$. In cases of surinfection local or systemic antibiotics are added. Adequate pain management is necessary because the pain often is intolerable. It is worthy to search for underlying diseases because in $50 \%$ of the cases there will be one which also needs to be treated.

Table 1. Systemic Therapy is Reviewed and Summarized [5, 13, 14]

\begin{tabular}{|c|c|c|}
\hline Drugs & Dose & Side Effects/Remarks \\
\hline Prednisone & $1-2 \mathrm{mg} / \mathrm{kg} / \mathrm{d}$ po & Beware of cardiac disease and diuretics \\
\hline Sulfa drugs & $\begin{array}{l}200 \mathrm{mg} / \mathrm{d} \text { (dapsone) } \\
1-4 \mathrm{~g} / \mathrm{d} \text { (sulfasalzine) }\end{array}$ & Control met-haemoglobin \\
\hline Minocycline & $200-300 \mathrm{mg} / \mathrm{d}$ & Teratogenic; Beware of sunburn \\
\hline Tacrolimus & $\begin{array}{l}0.05 \mathrm{mg} / \mathrm{kg} / \mathrm{d} \mathrm{IV} \\
0.15-0.30 \mathrm{mg} / \mathrm{kg} / \mathrm{d} \text { po }\end{array}$ & $\begin{array}{l}\text { Blurred vision, hepato- } \& \text { nephrotoxic, hypertension, } \\
\text { tremor; Beware of sunburn }\end{array}$ \\
\hline Tumor necrosis $\alpha$ inhibitor infliximab & $3-5 \mathrm{mg} / \mathrm{kg}$ IV at week $0,2,6$ and every 8 week thereafter & Beware of infection \& sunburn; Screen for tuberculosis \\
\hline Azathioprine & $1-2 \mathrm{mg} / \mathrm{kg} / \mathrm{d}$ po & $\begin{array}{l}\text { Good when IBD is associated; Beware of blood counts } \\
\text { and transaminases }\end{array}$ \\
\hline Mycophenolate mofetil & $2 \mathrm{~g} / \mathrm{d}$ & Renal and hepatic side effects; Beware of sepsis \\
\hline Etanercept & $2 \times 50 \mathrm{mg} /$ week & Screen for tuberculosis \\
\hline Thalidomide & $50-300 \mathrm{mg} / \mathrm{d}$ po & Teratogenic \\
\hline Leukocytapheresis & & Removal of white blood cells \\
\hline \multicolumn{3}{|l|}{ Hyperbaric oxygen therapy } \\
\hline Colchicine & & Gastro-intestinal side-effects \\
\hline
\end{tabular}


Long term outcome is still unpredictable. Relapses occur frequently, respectively in $70 \%$ of patients treated with prednisone and $66 \%$ of patients treated with cyclosporine A [16]. PG remains life-threatening with a mortality up to $30 \%$ in some series [5].

In this case we report a case of PG triggered by a surgical procedure in a breast cancer patient. It was noteworthy that the spread of redness and necrosis remained limited to the area that was exposed to her radiotherapy a year prior.

\section{CONCLUSION}

PG is diagnosed by exclusion but one should suspect it if a patient has a markedly painful ulcer with a bluish border which rapidly progresses. In $25-50 \%$ PG is provoked due to pathergy [4]. Standard wound care and antibiotics are ineffective. It is very important to rule out other possible causes by taking a biopsy and searching for underlying associated diseases. Typical is the rapid and dramatic response to corticosteroids [9], but if there is no response to treatment, the diagnosis should be reconsidered and the biopsy should be repeated [10].

\section{ABBREVIATIONS}

$\begin{array}{lll}\mathrm{IBD} & = & \text { Inflammatory bowel disease } \\ \mathrm{IV} & = & \text { Intravenous } \\ \mathrm{PG} & = & \text { Pyoderma gangrenosum } \\ \text { po } & = & \text { Per os, orally }\end{array}$

\section{REFERENCES}

[1] Brocq L. Nouvelle contribution a l'etude du phagedenisme geometrique. Ann Dermatol Syph 1916; 6: 1-39.
[2] Brunsting LA GWOPA. Pyoderma (ecthyma) gangrenosum: clinical and experimental observations in five cases occurring in adults. Arch Dermatol Syph 1930; 22: 655-80.

[3] Crowson AN, Mihm MC, Jr., Magro C. Pyoderma gangrenosum: a review. J Cutan Pathol 2003; 30: 97-107.

[4] Bennett ML, Jackson JM, Jorizzo JL, et al. Pyoderma gangrenosum. A comparison of typical and atypical forms with an emphasis on time to remission. Case review of 86 patients from 2 institutions. Medicine (Baltimore) 2000; 79: 37-46.

[5] Wollina U. Pyoderma gangrenosum--a review. Orphanet J Rare Dis 2007; 2: 19 .

[6] Banga F, Schuitemaker N, Meijer P. Pyoderma gangrenosum after caesarean section: a case report. Reprod Health 2006; 3 : 9.

[7] Sergent F, Joly P, Gravier A, Verspyck E, Marpeau L. [Pregnancy: a possible etiology of pyoderma gangrenosum. A case report and review of the literature]. J Gynecol Obstet Biol Reprod (Paris) 2002; 31: 506-11.

[8] Charles CA, Bialy TL, Falabella AF, et al. Poor prognosis of arthritis-associated pyoderma gangrenosum. Arch Dermatol 2004; 140: 861-4.

[9] Su WP, Davis MD, Weenig RH, Powell FC, Perry HO. Pyoderma gangrenosum: clinicopathologic correlation and proposed diagnostic criteria. Int J Dermatol 2004; 43: 790-800.

[10] Weenig RH, Davis MD, Dahl PR, Su WP. Skin ulcers misdiagnosed as pyoderma gangrenosum. N Engl J Med 2002; 347 1412-8.

[11] Hasselmann DO, Bens G, Tilgen W, Reichrath J. Pyoderma gangrenosum: clinical presentation and outcome in 18 cases and review of the literature. J Dtsch Dermatol Ges 2007; 5: 560-4.

[12] Powell FC, Su WP, Perry HO. Pyoderma gangrenosum: classification and management. J Am Acad Dermatol 1996; 34: 395-409.

[13] Bhat RM. Management of pyoderma gangrenosum--an update. Indian J Dermatol Venereol Leprol 2004; 70: 329-35.

[14] Wollina U. Clinical management of pyoderma gangrenosum. Am J Clin Dermatol 2002; 3: 149-58.

[15] Horner B, El Muttardi N, Mercer D. Pyoderma gangrenosum complicating bilateral breast reduction. Br J Plast Surg 2004; 57: $679-81$.

[16] Vidal D, Puig L, Gilaberte M, Alomar A. Review of 26 cases of classical pyoderma gangrenosum: clinical and therapeutic features. J Dermatolog Treat 2004; 15: 146-52. 